\title{
Crises in a driven Josephson junction studied by cell mapping
}

\author{
Sørensen, Mads Peter; Davidson, A.; Pedersen, Niels Falsig; Pagano, S.
}

Published in:

Physical Review A

Link to article, DOI:

10.1103/PhysRevA.38.5384

Publication date:

1988

Document Version

Publisher's PDF, also known as Version of record

Link back to DTU Orbit

Citation (APA):

Sørensen, M. P., Davidson, A., Pedersen, N. F., \& Pagano, S. (1988). Crises in a driven Josephson junction studied by cell mapping. Physical Review A, 38(10), 5384-5390. https://doi.org/10.1103/PhysRevA.38.5384

\section{General rights}

Copyright and moral rights for the publications made accessible in the public portal are retained by the authors and/or other copyright owners and it is a condition of accessing publications that users recognise and abide by the legal requirements associated with these rights.

- Users may download and print one copy of any publication from the public portal for the purpose of private study or research.

- You may not further distribute the material or use it for any profit-making activity or commercial gain

- You may freely distribute the URL identifying the publication in the public portal

If you believe that this document breaches copyright please contact us providing details, and we will remove access to the work immediately and investigate your claim. 


\title{
Crises in a driven Josephson junction studied by cell mapping
}

\author{
M. P. Soerensen \\ Laboratory of Applied Mathematical Physics, The Technical University of Denmark, DK 2800 Lyngby, Denmark \\ A. Davidson \\ IBM Research Division, Thomas J. Watson Research Center, Yorktown Heights, New York 10598 \\ N. F. Pedersen \\ Physics Laboratory I, The Technical University of Denmark, DK 2800 Lyngby, Denmark \\ S. Pagano \\ Laboratory of Applied Mathematical Physics, The Technical University of Denmark, DK 2800 Lyngby, Denmark
}

(Received 16 May 1988)

\begin{abstract}
We use the method of cell-to-cell mapping to locate attractors, basins, and saddle nodes in the phase plane of a driven Josephson junction. The cell-mapping method is discussed in some detail, emphasizing its ability to provide a global view of the phase plane. Our computations confirm the existence of a previously reported interior crisis. In addition, we observe a boundary crisis for a small shift in one parameter. The cell-mapping method allows us to show both crises explicitly in the phase plane, at low computational cost.
\end{abstract}

\section{INTRODUCTION}

Strange attractors of a periodically driven Josephson junction are studied numerically by means of simple cell mapping. ${ }^{1}$ We focus here on the sudden changes of strange attractors, which may occur when some parameter values are altered. These changes are called crises. ${ }^{2-5}$ Two different types of crises are discussed, namely, the boundary crisis and the interior crisis.

Following the notation of Grebogi et al..$^{2,3}$ we call the collision of a stable chaotic attractor with an unstable periodic orbit at a basin boundary, a boundary crisis. Boundary crises result in the sudden annihilation of the chaotic attractor. In contrast, an interior crisis arises from a similar collision of attractors from within a single basin. In this case the chaotic attractor has a sudden expansion in phase space.

We have chosen to study the driven Josephson junction for a range of parameters already studied by Kautz. ${ }^{5,6}$ This parameter range is characterized by hysteretic switching between attractors. Such behavior is known ${ }^{7}$ to exclude significant noninteger phase locking and "the devil's staircase" is not considered here. Kautz has found two crises, one at each end of the parameter range, and he has identified one as an interior crisis. We confirm this result, and show that the other crisis is a boundary crisis.

To study numerically the global behavior of the Josephson junction it is advantageous to use cell-mapping methods. ${ }^{1,8-11}$ We shall use only the simple cellmapping algorithm where the two-dimensional phase space is divided into rectangles or cells and the governing dynamical equation defines a way to map each cell onto another in the phase plane. A more elaborate cell- mapping method is the generalized cell-mapping procedure. ${ }^{9}$ Here it is allowed for a mapping of a cell to have multiple image cells with appropriate individual mapping probabilities. In Ref. 10 the two above cellmapping methods are combined into "compatible simple" and "generalized" cell mapping.

The organization of this paper is as follows. Section II briefly introduces the dynamical equation of the system under study, including parameter values. Then the concepts of the cell-mapping method are developed, and some of its limitations are pointed out. Section III presents the computed current-voltage $(I-V)$ curve for the parameters of interest, along with a one-dimensional Poincare map. These were the tools used by Kautz in his investigation, and we repeat them here both as confirmation of his work, and to provide benchmarks for our cell-mapping study. Section IV uses cell-mapping and Poincaré sections to identify parameters for which a boundary crisis occurs, and to locate in phase space the relevant attractors. Section $\mathrm{V}$ extends cell mapping to compute basins of attraction, and manifold crossings. Both the new boundary crisis identified in Sec. IV and Kautz's interior crises are studied.

\section{MODEL EQUATION AND NUMERICAL METHOD}

The equation describing the dynamics of a driven Josephson junction is given by ${ }^{12}$

$$
\ddot{\phi}+\alpha \dot{\phi}+\sin \phi=\eta+A \sin (\omega t) \text {. }
$$

Here overdots denote derivatives with respect to time $t$. $\phi=\phi(t)$ is the phase difference across the junction, $\alpha$ is the quasiparticle damping term, given by $\alpha=1 / \sqrt{\beta}$, where $\beta$ is the McCumber parameter, ${ }^{12}$ and $\eta$ is the con- 
stant dc bias current. $A$ and $\omega$ denote the amplitude and frequency of the external driving force, respectively. Only the $\eta$ term has been varied and the other parameters have been fixed at the values $\alpha=0.2, A=10.198039$ (for comparison with Ref. 5), and $\omega=1.0$. The dynamical equation (1) is solved numerically using a fourth-order Runge-Kutta algorithm. The solution may be displayed in the phase plane as a Poincare section where points $(\phi(t), \dot{\phi}(t))$ are plotted after every period $T=2 \pi / \omega$ of the drive cycle. Here $\phi(t)$ is treated $\bmod 2 \pi$, from $-\pi$ to $\pi$. The Poincare map gives a good geometrical view of the dynamics by which we can identify the different attractors. To study the global behavior of the system, the basins of attraction of the different attractors must be found. To do this by solving Eq. (1) numerically with initial conditions distributed uniformly over a finite subset of the phase plane is a very time consuming procedure. A more efficient way of getting the basins of attraction is to use the cell-mapping methods. ${ }^{1,8-11}$

In the cell-mapping method we divide an interesting subset of the phase plane into cells. Let us for simplicitly introduce the notation $\phi=x_{1}$ and $\dot{\phi}=x_{2}$. A finite rectangular subset of the two-dimensional phase space of Eq. (1) is then divided into $N$ rectangular cells. We shall use uniform cells of width $h_{1}$ and height $h_{2}$. If $N_{1}$ denotes the number of cells in the $x_{1}$ direction, and $L_{1}$ denotes the length of the subset in the $x_{1}$ direction, then $h_{1}=L_{1} / N_{1}$. Similarly, we have for the $x_{2}$ direction that $h_{2}=L_{2} / N_{2}$. The cells are counted sequentially, beginning, for example, at the cell in lowest row to the left, counting along the lowest row, and ending with the cell No. $N=N_{1} N_{2}$ in the uppermost row to the right. The $i$ th cell $Z^{i}=\left(z_{1}^{i}, z_{2}^{i}\right), i=1,2, \ldots, N$, is now defined to contain all points $\left(x_{1}, x_{2}\right)$ that satisfy

$$
z_{j}^{i}-\frac{1}{2} h_{j} \leq x_{j}<z_{j}^{i}+\frac{1}{2} h_{j}, \quad j=1,2 .
$$

$z_{j}^{i}, j=1,2$ are the coordinates of the center point of the $i$ th cell. To get a cell to cell mapping we integrate Eq. (1) over one period $T$ of the external driving force using the center point $Z^{i}$ of the $i$ th cell as the initial condition. This is done for each cell $i$. The final-state point after the integration will lie either inside an existing cell which can be identified according to Eq. (2) or outside the subset of interest. In the later case we shall introduce a sink cell, denoted $Z^{0}$, which then absorbs points escaping outside the region of interest.

By definition the sink cell is mapped into itself. To each cell, including the sink cell, we have now assigned an image cell and the mapping so defined is denoted by $C$. Let $Z(1)$ be an arbitrary initial cell. After $n$ applications of $C, Z(1)$ is mapped into $Z(n)$ and we write $Z(n+1)=C(Z(n))$. The evolution of the system can be periodic in the sense that a cell $Z^{*}$ is mapped into itself after $K$ applications of $C$. Such a motion is called $K$ periodic or a $P-K$ motion. The $K$ different cells traversed in a $P$ - $K$ motion form a periodic group and the cell belonging to this group are called $P-K$ cells. The cells which eventually are mapped into this group through repeated application of $C$ belong to the basin of attraction of this $P-K$ group.
The evolution of the system from a given initial cell can lead to only three different outcomes: (1) The initial cell is itself a periodic cell, (2) the initial cell belongs to the basin of attraction of a $P-K$ group, or (3) the initial cell is mapped into the sink cell. This elementary analysis forms the basis for a very efficient unraveling numerical algorithm designed by $\mathrm{Hsu}$ and Guttalu in Ref. 1 . This algorithm has been used to determine all regions of attraction and their basins of attraction of Eq. (1) in a given subset of phase space. After having made the discretization into cells and determined the cell-to-cell map, the system dynamics have been reduced to a simple sorting of integers which requires only a minimum amount of computer resources. However, the simple cell-mapping procedure has to be used cautiously. The reason is that the discretization introduces errors which result in qualitative differences from the original system. The cell map may find periodic groups which correspond to unstable attractors in the continuous problem. Some periodic groups with a high period may correspond to a chaotic attractor, or to a quasiperiodic attractor. Several groups may represent different parts of the same attractor in the original system. These errors can be ameliorated by using finer grids or by using the refined cell-mapping procedures described in Refs. 9 and 10, but they are inherent in the discretization of phase space used in cell mapping.

\section{INTERPRETATION OF THE $I$ - $V$ CHARACTERISTIC IN TERMS OF CRISES}

Before applying the cell-mapping procedure, we shall give an interpretation in terms of crises of the part of the $I-V$ characteristic shown in Fig. 1. This $I-V$ curve was obtained by numerically solving Eq. (1) and plotting the bias current $\eta$ versus the time average $\langle\dot{\phi}\rangle$, which is proportional to the voltage across the junction. The same $I$ $V$ curve (with the same parameters) has been published by $\mathrm{Kautz}^{5,6}$ in another connection, hence a direct comparison is possible.

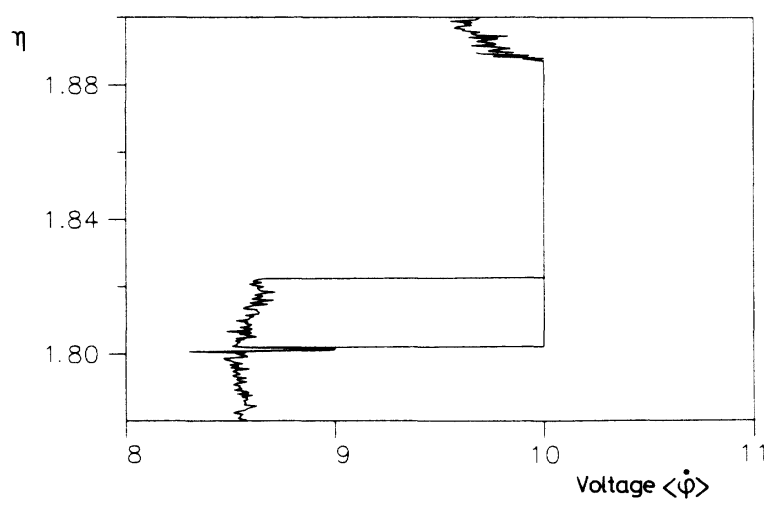

FIG. 1. $I-V$ characteristic for the parameter values $\alpha=0.2$, $A=10.198039027$, and $\omega=1.0$. Note that all quantities in all figures are in dimensionless units, using normalizations as explained in Ref. 12. 
In Fig. $1 \eta$ was varied from $\eta=1.78$ to 1.90 , and the other parameters were fixed at the values stated in Sec. II. We focus on the vertical line segment in Fig. 1 at the value 10 on the voltage axis. It can be shown that along this line $\phi$ is phase locked to the oscillatory driving force. The question we address (and which is addressed by $\mathrm{Kautz}^{5}$ ) is, how is the phase lock destroyed at either end of this constant voltage step? Kautz was able to show that the gradual departure at the top of the step is due to an interior crisis, but he left the lower end unresolved. Note that the lower end involves a hysteresis loop, where the voltage jumps back and forth between the step and some noisy curve as the current is changed very slightly between $\eta=1.802$ and 1.822 . The hysteresis loop implies that two attractors coexist in phase space, and suggests that a boundary crisis between these two attractors could be involved.

This is shown more clearly in Fig. 2, where we have plotted $\dot{\phi}(n T)$ from the Poincaré section at corresponding $\eta$ values, where $T=2 \pi / \omega$ denotes the period of the external driving frequency. From Fig. 2 it is evident that a chaotic dynamic state exists in the $\eta$ interval $1.78<\eta<1.822$ and this chaotic state causes the erratic form of the $I-V$ characteristic.

Coexistent with the chaotic dynamic state there is a simple period-one solution in the $\eta$ interval $1.802<\eta<1.822$. The associated piece of the $I-V$ curve is phase locked and the voltage $V=\langle\dot{\phi}\rangle=10$ is strictly constant. The phase-locked state is characterized by the relation $\phi(t+n T)=\phi(t)+2 m \pi$ and accordingly the voltage across the junction becomes $V=\langle\dot{\phi}\rangle=(\mathrm{m} /$ $n)(2 \pi / T)$. In the case of Fig. $1 m=10$ and $n=1$, i.e.,

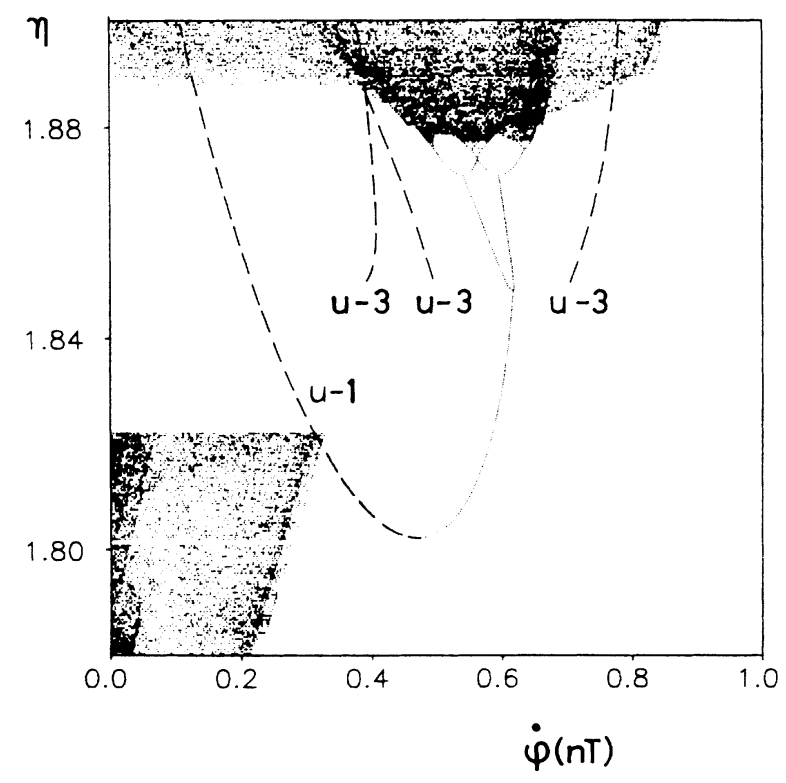

FIG. 2. Bifurcation diagram showing a boundary crisis and an interior crisis. The parameter values used are as in Fig. 1. The unstable period-one and period-three saddles are shown as dashed curves marked $u-1$ and $u-3$, respectively. the junction is locked on the step No. $m / n=10$. When $\eta$ is increased beyond 1.822 the chaotic attractor disappears and only the period-one or phase-locked state remains. The reason for this behavior is apparent from Fig. 2. Together with the stable period-one state there exists an unstable period one hyperbolic point shown as a dashed curve and marked $u-1$. The unstable periodic points shown in this figure and in Figs. 4-7 have been located with the Newton-Raphson algorithm described in Ref. 13. When the chaotic attractor collides with this unstable period solution, a boundary crisis occurs and the chaotic attractor disappears. After the occurrence of this boundary crisis only the phase locked part of the $I-V$ curve remains, as may be seen in Fig. 1.

The interior crisis at the top of the voltage step in Fig. 1 can also be discerned in Fig. 2. Here we see a Feigenbaum sequence with period-doubling bifurcations when increasing $\eta$ from 1.822 up to 1.888 . At the first perioddoubling bifurcation we have $m=20$ and $n=2$, at the next we have $m=40$ and $n=4$, and so on. This means that the junction remains on the phase-locked step No. $m / n=10$, and even in the chaotic region, which follows the period-doubling bifurcations, the voltage across the junction remains constant. As the voltage is determined as a mean value $\langle\dot{\phi}\rangle$, we cannot see from the $I-V$ curve alone that the above period-doubling bifurcation followed by a chaotic window has occurred. However, at $\eta=1.888$ a change in the $I-V$ curve is again observed. The voltage $V$ decreases and becomes irregular. From the Poincare section we notice that an unstable periodthree orbit marked $u-3$ and created at $\eta=1.851$, has collided with the chaotic region mentioned above. This collision results in an interior crisis and accordingly the chaotic region expands suddenly, filling out a larger subset of phase space. When this expansion occurs the junction is no longer phase locked on step No. 10.

\section{CELL-TO-CELL MAPPING AND BOUNDARY CRISIS}

We shall now apply the cell-to-cell mapping to show in more detail the collision between the unstable periodic orbit and the chaotic attractor in the $\eta$ interval [1.80;1.825]. Figures $3(a)-3(c)$ are phase plane plots approaching the crisis, and in Fig. 3(d), the crisis has occurred. All of these plots show the conventionally generated Poincaré maps as a multitude of tiny dots, one dot for each cycle of the periodic driving force. The larger symbols represent various periodic groups as found by the cell-mapping method. The small.open circle labeled $u-1$ is a saddle point as calculated by the NewtonRaphson technique. ${ }^{13}$ No basins of attraction are shown. The extended chaotic attractor made visible by the Poincaré maps in Figs. 3(a)-3(c) disappears in Fig. 3(d) because of the crisis.

The general trend to follow in these figures is that as $\eta$ rises and we move from Fig. 3(a) to Fig. 3(b) in Fig. 3(c), a finger of the chaotic attractor moves toward the unstable saddle point. When the finger touches the saddle point the crisis occurs, and the chaotic attractor disappears, as in Fig. 3(d). The beauty of the cell-mapping method is, 
first, that it can find unstable attractors, and second, that it gives us a measure of confidence that we are following all the relevant attractors in phase space.

In the cell-to-cell mapping we have divided the subset $\left[-\pi \leq x_{1} \leq \pi\right] \times\left[-2 \pi \leq x_{2} \leq 2 \pi\right]$ into $N X \times N Y=50$ $\times 100$ cells. In Fig. 3(a) $\eta$ equals 1.80 and only one chaotic attractor exists in this $\eta$ value. However, in the cell-to-cell map we found five periodic groups. For each of these groups the average voltage was calculated for the associated periodic motion and the attractors were then sorted according to their voltages. In the original system the voltage corresponding to a given attractor is an integer if biased on a phase-locked state. To each phaselocked state there exists only one attractor. Therefore, when using cell mapping, we regard two or more attractors which are formally distinct as identical, provided their periodic motion gives rise to the same integer voltage. In regions of chaos the chaotic attractor will result in voltages which are nonintegers.

The identification of chaotic attractors with periodic groups is a little more subtle. Due to the finite number of cells, we can only get periodic orbits when using cell mapping. However, we may identify a chaotic attractor with a periodic group if the group period is large and the corresponding voltage is noninteger. As in the phaselocked case, formally distinct cell-map groups will be considered to belong to the chaotic attractor, provided the voltage is noninteger (this will be the case even if the voltages are not identical). The only requirement is that the voltages are noninteger and that the Poincare map reveals only one chaotic attractor for the parameter values under consideration.

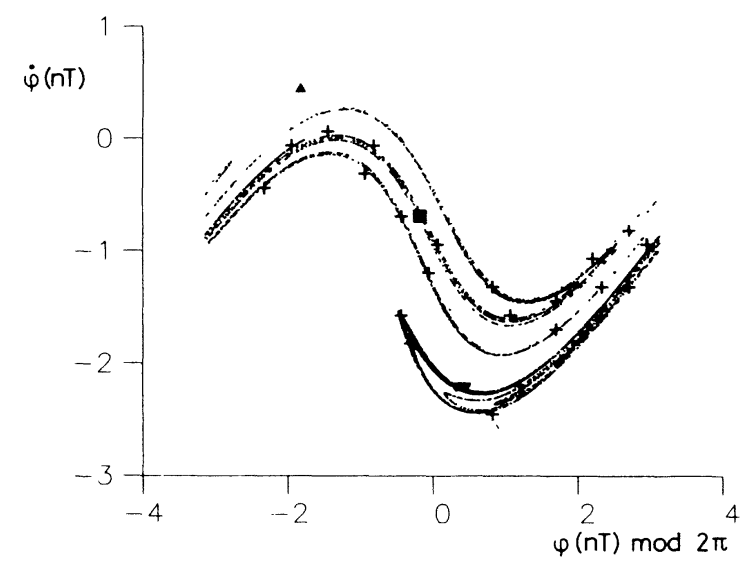

(a)

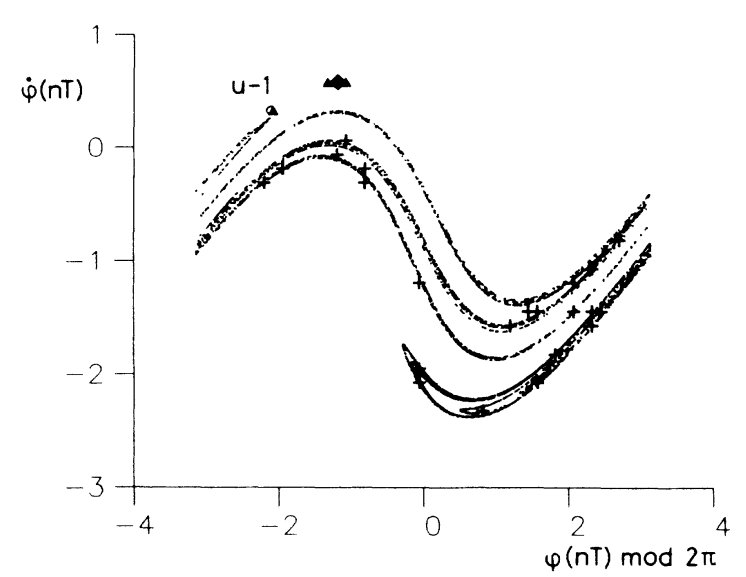

(c)

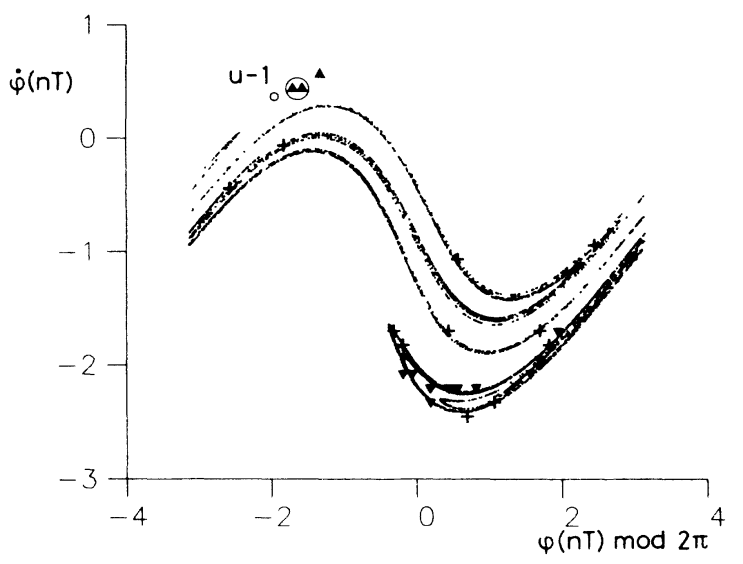

(b)

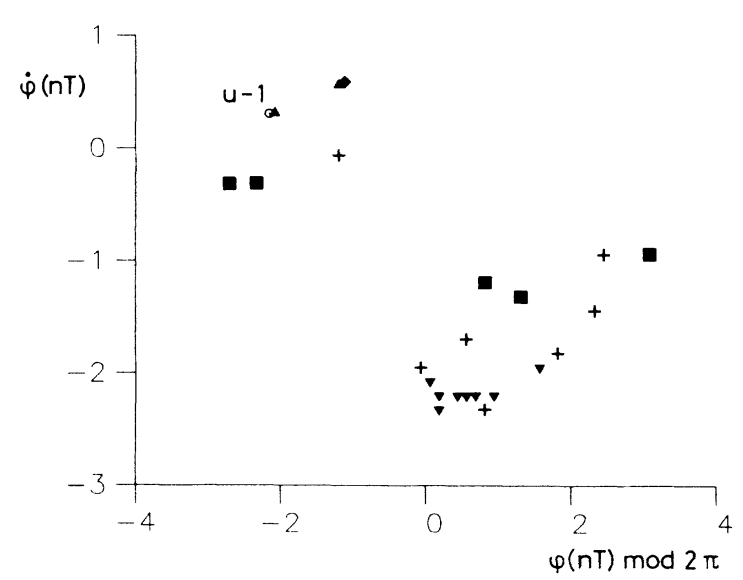

(d)

FIG. 3. Poincaré section of the chaotic attractor for Eq. (1) at parameter values $\alpha=0.2, A=10.198039027$, and $\omega=1.0$. Periodic attractors obtained from the cell-mapping procedure are also shown using the subset $\left[-\pi \leq x_{1} \leq \pi\right] \times\left[-2 \pi \leq x_{2} \leq 2 \pi\right]$ which have been divided into $50 \times 100$ cells. The average voltage of the periodic groups marked by down-pointing triangles, squares, and uppointing triangles, are 8,9 , and 10 , respectively, corresponding to step Nos. 8,9 , and 10 . The crosses denote periodic groups with noninteger voltage. Stable periodic orbits determined directly from the Poincare section are marked with a diamond and circles show period-one saddles determined from the Newton-Raphson algorithm. (a) $\eta=1.80$, (b) $\eta=1.81$, (c) $\eta=1.82$, (d) $\eta=1.825$. 
As mentioned above we found five groups in the cellto-cell map at $\eta=1.80$ [see Fig. 3(a)]. Two of these are a $P-16$ group with voltage $V=8.750$ and a $P-6$ group with voltage $V=8.501$. As the voltages are noninteger and as we know from the Figs. 1 and 2 that only one chaotic attractor exists at $\eta=1.80$ the two periodic groups are artificially distinct and we shall regard both of them as belonging to the chaotic attractor. In Fig. 3(a) these two groups together are marked by crosses, and we observe that they fit well to the real attractor obtained from the Poincaré section. This means that the cell-to-cell map provides a fairly accurate picture of the chaotic attractor even with such a crude discretization grid as $50 \times 100$. Furthermore, the cell map reveals a $P-2$ group, marked by downward pointing triangles, with voltage $V=8.000$ (step 8), a $P-1$ group marked by a solid square with $V=9.008$ (step 9), and finally a $P-1$ group marked by an upward pointing triangle with $V=10.003$ (step 10). These periodic cell-map groups have been found artificially, and they are not stable in the original model equation (1). However, the above three steps or attractors are stable for $\eta$ values nearby. We believe that there are periodic transients or "shadows" of the above periods which the cell-map procedure picks up. This fact is useful when searching for attractors in a given parameter region.

In Fig. 3(b) $\eta$ is raised from 1.80 to 1.81 and from Figs. 1 and 2 two coexistent attractors are present, a chaotic one and a periodic one with $V=10$. Six periodic groups have been found by cell mapping. There is a $P-12$ group with $V=8.498$, marked by crosses, which is clearly associated with the chaotic attractor. Three other groups, marked in common with down pointing triangles are associated with a shadow of a nearby $V=8$ attractor. Finally cell mapping produced two $V=10$ attractors, in the form of a $P-1$ group and a $P-2$ group marked by up pointing triangles in Fig. 3(b). The two circled triangles are the $P$-2 group.

Clearly, these last two groups correspond to step No. 10 in the continuous problem, and are not shadows. The $P$-1 group coincides with the period-one orbit [marked by a diamond in Fig. 3(c)] obtained from direct numerical simulations of Eq. (1). The $P-2$ group is the unstable periodic orbit created at the saddle-node bifurcation at $\eta=1.802$. It is easy to understand why the cell map is able to pick up this unstable saddle point. If the saddle point is approached along one of its two stable manifolds it will take a very long time to reach the saddle point. Therefore a cell around the saddle or very close to it may be mapped into itself. In this case two cells close to the saddle have been mapped into themselves and their positions indicate with good accuracy the position of the saddle point. This has been verified by the Newton-Raphson iteration procedure for finding fixed points. ${ }^{13}$ The exact position of the unstable saddle point is shown by the small open circle in Fig. 3(b).

In Fig. 3(c) $\eta$ is raised to the value 1.82. The cell-map procedure gives three periodic groups on step No. 10, namely, two $P-1$ groups and one $P-2$ group. One of the $P-1$ groups is positioned at the unstable orbit and in Fig. 3 (c) its position is marked by the lettering $u-1$. We ob- serve that the chaotic attractor (small dots) nearly touches the saddle point $u-1$, so that we are close to the boundary crisis which occurs at $\eta=1.822$. The stable periodic orbit determined directly from Eq. (1) is marked by a diamond, and agrees well with the cell-map result. Similarly the Newton-Raphson algorithm finds the unstable saddle point to be located very close to the unstable point found by cell mapping. In Fig. 3(c) the exact position is shown by a circle. Furthermore, the cell-to-cell map shows a $P-22$ group with $V=8.634$ and again this large group fits well with the chaotic attractor obtained from the Poincaré section.

In Fig. 3(d) $\eta$ has been increased to the value 1.825 which is above the value of $\eta=1.822$ where the boundary crisis occurs. The chaotic attractor has vanished and only the period-one stable solution marked by a diamond is present. However, the cell-map procedure has found six periodic groups. Two of them, marked with up triangles, correspond to step No. 10, and the one closest to the diamond mark is the stable period-one solution and the other one is the unstable saddle point, still labelled $u-1$, which has just collided with the chaotic attractor. The position of the unstable point found from the NewtonRaphson iteration procedure is marked by a circle and again the cell-map result shows excellent agreement with the Newton-Raphson algorithm. The other groups that the cell-to-cell map has picked are again shadows, the results of transients of real attractors which appear close to the $\eta$ value of 1.825 . These artificial groups are the following: one $P-5$ group with voltage $V=9.000$, a $P-6$ and a $P$-2 group with voltages $V=8.004$ and $V=7.996$, respectively, and finally a "chaotic" $P-7$ group with $V=8.433$. This $P-7$ group is the result of the chaotic transient due to the chaotic attractor which existed before the boundary crisis.

Figure 3 clearly shows that a crisis occurs as the strange attractor touches the saddle point, but why should such an event be termed a boundary crisis? The reason is, as shown in Sec. $\mathrm{V}$ that the saddle point lies directly on a basin boundary.

\section{BASIN BOUNDARIES AND HETEROCLINIC MANIFOLD CROSSINGS}

In this section we shall use the cell-to-cell mapping to illustrate heteroclinic tangencies and crossings of the stable and unstable manifolds of saddle orbits on basin boundaries. 2,3,14 Both the boundary crisis and the interior crisis will be treated. We will use cell mapping to compute basins of attraction, and illustrate that the saddle points lie on basin boundaries. We consider the subset $\left[-\pi \leq x_{1} \leq \pi\right] \times\left[0 \leq x_{2} \leq 1\right]$ which is divided into $N X \times N Y=100 \times 100$ cells. Note that the division into cells is finer in this case then previously; therefore, the cell-to-cell map will give considerably more precise results. We start with the boundary crisis. Figure 4 shows the basin of attraction (marked by dots) of the stable period-one solution on step No. 10 , just at the $\eta$ value 1.822 where the boundary crisis occurs. Note that the chaotic attractor just touches the border of the basin of attraction for the No. 10 step. This "touching" is a 


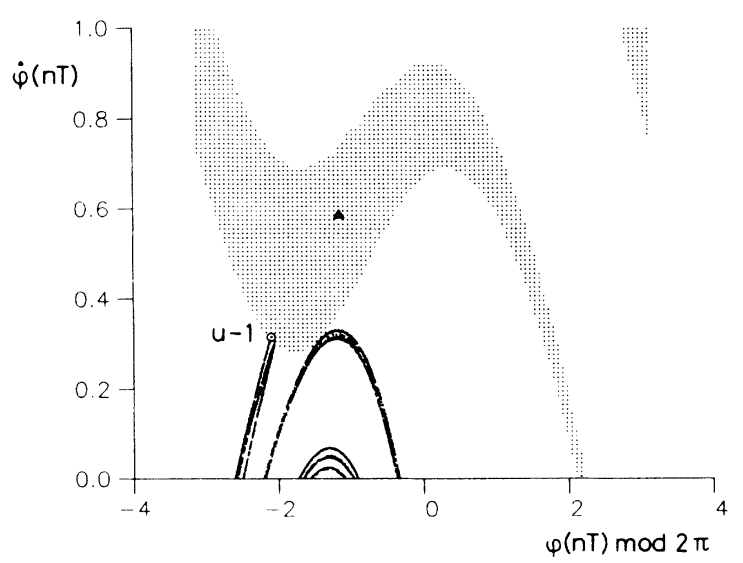

FIG. 4. Heteroclinic tangencies at the boundary crisis. $\eta=1.822$, and the other parameter values used are as in Fig. 1. The basin of attraction for the period-one solution (marked by up-pointing triangles) is shown as small dots. The circle shows the position of the unstable period-one saddle point $u-1$.

tangency, as discussed below.

The attracting point is marked by the up triangle. The white region consists of cells which disappear from the subset of interest and they are mapped into the sink cell. (Considering all of the phase plane, points in the white region actually belong to the basin of attraction of the chaotic attractor.) The period-one saddle point $(u-1)$ is marked by a circle in Fig. 4 and lies on the borderline of the basin of attraction of the stable period-one attractor. This borderline is also the stable manifold of the saddle point $u-1$. That is, points on the basin boundary are attracted to $u-1$. One branch of the unstable manifold (along which points are repelled from $u$ - 1 , is directed along the chaotic attractor. The other unstable branch extends toward the up triangle. We see from Fig. 4 that part of the unstable manifold, namely, the strange attractor, actually touches the stable manifold, the basin boundary, in several places at the same time. Hence if the system begins in a state of chaos on the strange attractor, it will eventually touch the stable maniold of $u-1$ and be sent to the stable fixed point attractor with $V=10$ represented by the up triangle in Fig. 4, and this is the most complete illustration of the boundary crisis.

From Fig. 4 this tangency of the strange attractor at $u-1$ would be called a homoclinic tangency, since only one saddle point is shown. However, since the mapping was computed $\bmod 2 \pi$, we actually have infinitely many saddle points. In accordance with Ref. 2 , we believe that the unstable manifold of a given saddle accumulates on the stable manifold of the previous saddle point, and accordingly Fig. 4 shows heteroclinic tangencies when the boundary crisis occurs.

In Fig. 5, $\eta$ has been increased to the value 1.823 that is above the value where the boundary crisis occurred. The basin of attraction of the period-one attractor, shown as dots, has been obtained using the cell-to-cell mapping procedure. The crosses denote periodic groups that lie on the transient chaotic attractor which is a remnant of

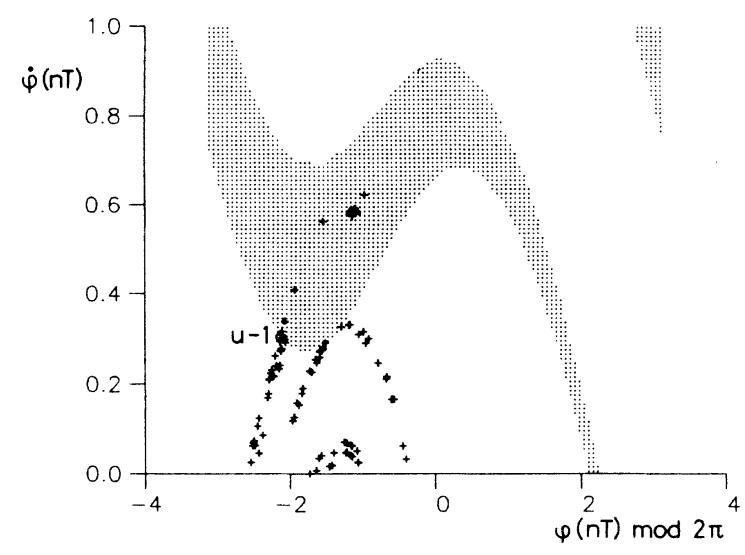

FIG. 5. Heteroclinic crossings after the boundary crisis. $\eta=1.823$, and the other parameter values used are as in Fig. 1 . Notation as in Fig. 4.

the stable chaotic attractor for $\eta$ less than 1.822. This transient indicates heteroclinic crossings of stable and unstable manifolds. Points on the transient chaotic attractor will eventually come close to the saddle point, marked by the circle in Fig. 5, passing it along the unstable orbit which lies in the basin of attraction of the stable periodone attractor. Thereby, the chaotic attractor from Fig. 4 is destroyed (that is, the attractor for the No. 10 step, marked by the up triangle, is now the only stable attractor).

Now we consider the interior crisis. Fig. 6 depicts the phase-locked chaotic attractor (solid line) as obtained from direct numerical simulation of Eq. (1) at $\eta=1.886$. This chaotic attractor appears after the Feigenbaum sequence seen in Fig. 2. The cell-to-cell map found a $P-17$ group with $V=10.000$, a $P-1$ group with $V=9.996$, and finally a $P$-2 group with $V=10.001$. These three cellmap groups all belong to step No. 10. The associated

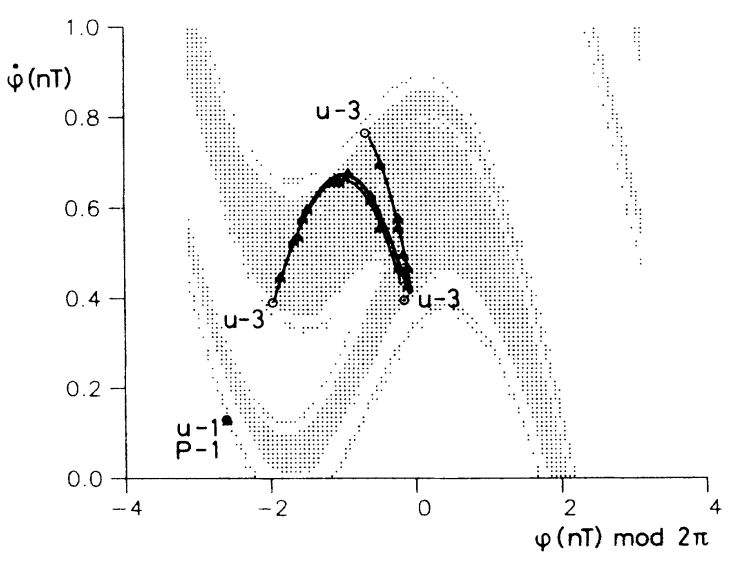

FIG. 6. Heteroclinic tangencies at the interior crisis. $\eta=1.886$, and the other parameter values used are as in Fig. 1 . Notation as in Fig. 4. 


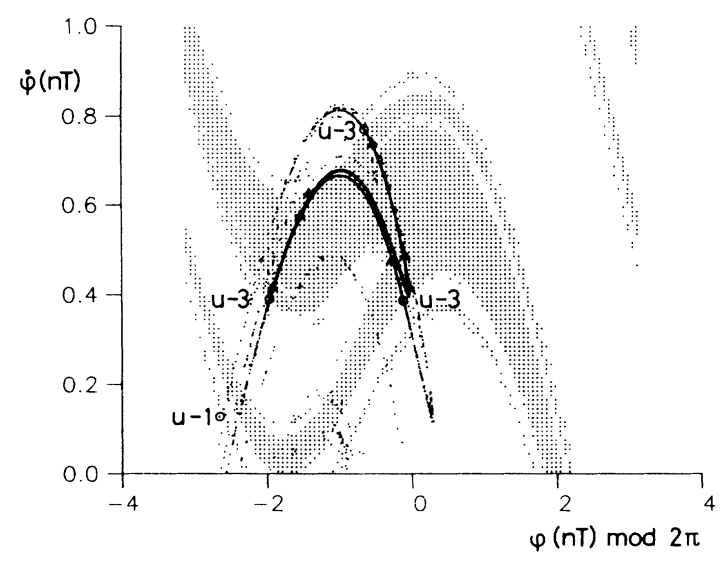

FIG. 7. Heteroclinic crossings at the interior crisis. $\eta=1.888$, and the other parameter values used are as in Fig. 1. Notation as in Fig. 4.

basin of attraction for these three attractors is shown as dots. The cell-map groups are marked by up triangles, and they all lie, except for the $P$-1 group, exactly on the phase-locked chaotic attractor (solid line). From the Newton-Raphson algorithm we have identified the $P-1$ group as the period-one saddle point shown in Fig. 2 and marked by a circle in Fig. 6. The interior crisis occurs between $\eta=1.886$ and $\gamma=1.887$ and what happens is that the chaotic attractor collides with the unstable period-three saddle ${ }^{5}$ shown as circles and labelled $u-3$ in Fig. 6. The period-three saddle has been obtained by using the Newton-Raphson algorithm. Its trajectories are shown as dashed curves in Fig. 2 (marked again by the lettering $u$-3) which also indicates the collision between the chaotic attractor and the period-three saddle. The chaotic attractor is again a subset of the unstable manifolds of the period-three saddles. The stable manifolds lie on the basin boundaries. From Fig. 6 we observe that the unstable manifolds become tangent to the stable manifolds at the $\eta$ value where the interior crisis happens.
For $\eta$ above this value the unstable and stable manifolds intersect each other resulting in an expansion of the chaotic attractor. This intersection is apparent from Fig. 7 where $\eta$ has been increased to 1.888 .

\section{CONCLUSIONS}

We have used cell-to-cell mapping to study and explicitly display a boundary crisis and an interior crisis in a driven Josephson junction. Using a coarse cell division the cell-mapping procedure will pick up remnant periodic and remnant chaotic transients in addition to the original existing attractors. With finer cell division we obtained very precise pictures of the tangencies and crossings of unstable and stable manifolds during both a boundary crisis and an interior crisis. This has been confirmed by calculating fixed points from the Newton-Raphson algorithm and from Poincare sections.

When exploring the global behavior of the Josephson junction the cell-mapping procedure provides a fairly accurate picture of the dynamics. Stable and unstable fixed points and associated basins of attractions can be found efficiently and at a low computational cost. The same applies to the strange attractors and their basins of attraction. When more detailed and accurate results are needed one can use the information gained from the cell mapping in applying the more precise tools, such as the Poincaré map and the Newton-Raphson iteration procedure. This is particularly valuable in the latter case where a good initial guess for the position of an unstable fixed point is necessary.

\section{ACKNOWLEDGMENTS}

We gratefully acknowledge financial support from the European Research Office of the U.S. Army through Contract No. DAJA-37-82-C-0057, from IBM Denmark, and from the European Economic Community through Contract No. St 2-0267-J-C(A). We should also like to thank Henrik Svensmark for illuminating discussions.
${ }^{1}$ C. S. Hsu, Cell-to-Cell Mapping (Springer-Verlag, Berlin, 1987), and references therein; in particular, C. S. Hsu and R. S. Guttalu, J. Appl. Mech. 47, 940 (1980).

${ }^{2}$ C. Grebogi, E. Ott, and J. A. Yorke, Phys. Rev. Lett. 48, 1507 (1982).

${ }^{3}$ C. Grebogi, E. Ott, and J. A. Yorke, Physica 7D, 181 (1983).

${ }^{4}$ Yao Huang Kao, Jeun Chyuan Huang, and Yih Shun Gou, Phys. Rev. A 34, 1628 (1986).

${ }^{5}$ R. L. Kautz, in The Proceedings of the Workshop on Structure, Coherence, and Chaos in Dynamical Systems, Lyngby, Denmark, 1986, edited by P. L. Christiansen and R. D. Parmentier (Manchester University Press, Manchester, in press).
${ }^{6}$ R. L. Kautz, J. Appl. Phys. 52, 6241 (1981).

${ }^{7}$ P. Alstrom and M. T. Levinson, Phys. Rev. B 31, 2753 (1985).

${ }^{8}$ C. S. Hsu, J. Appl. Mech. 47, 931 (1980).

${ }^{9}$ C. S. Hsu, J. Appl. Mech. 48, 634 (1981).

${ }^{10}$ C. S. Hsu and H. M. Chiu, J. Appl. Mech. 53, 695 (1986).

${ }^{11}$ A. Davidson and N. F. Pederson, Phys. Rev. A 36, 2455 (1987).

${ }^{12}$ A. Barone and G. Paterno, Physics and Applications of the Josephson Effect (Wiley-Interscience, New York, 1982).

${ }^{13}$ T. S. Parker and L. O. Chua, Proc. IEEE 75, 982 (1987), special issue on chaotic systems.

${ }^{14}$ C. Grebogi, E. Ott, and J. A. Yorke, Physica 24D, 243 (1987). 


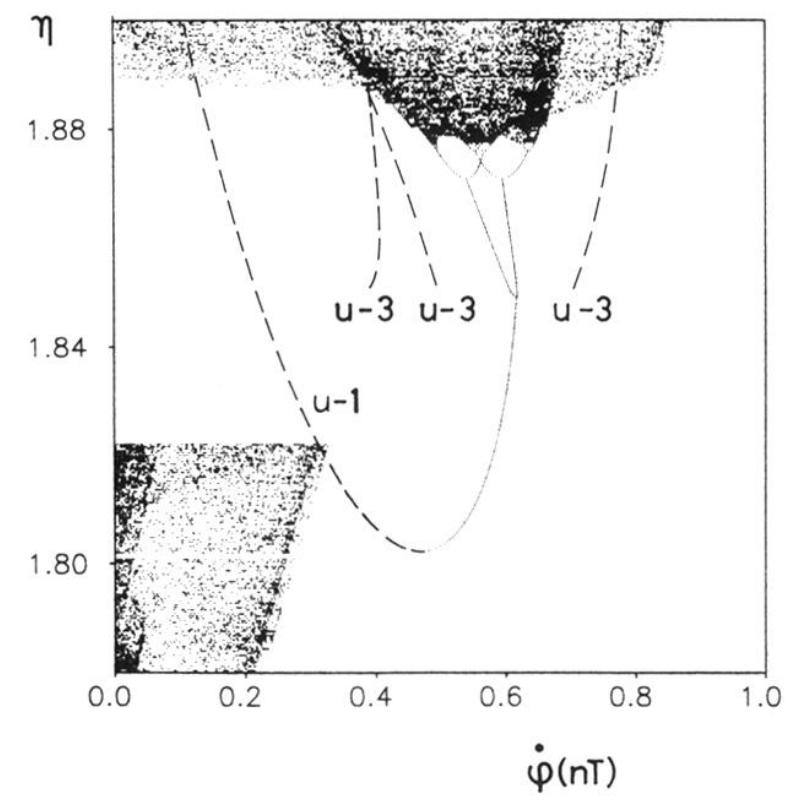

FIG. 2. Bifurcation diagram showing a boundary crisis and an interior crisis. The parameter values used are as in Fig. 1. The unstable period-one and period-three saddles are shown as dashed curves marked $u-1$ and $u-3$, respectively. 


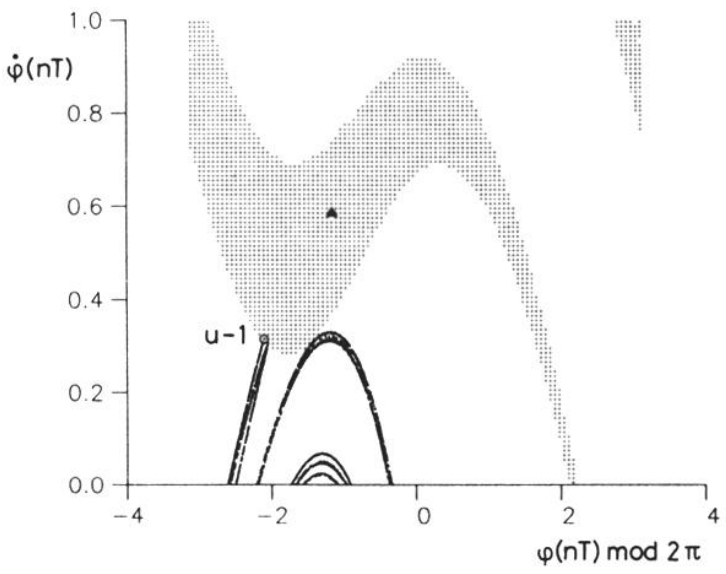

FIG. 4. Heteroclinic tangencies at the boundary crisis. $\eta=1.822$, and the other parameter values used are as in Fig. 1 . The basin of attraction for the period-one solution (marked by up-pointing triangles) is shown as small dots. The circle shows the position of the unstable period-one saddle point $u$ - 1 . 


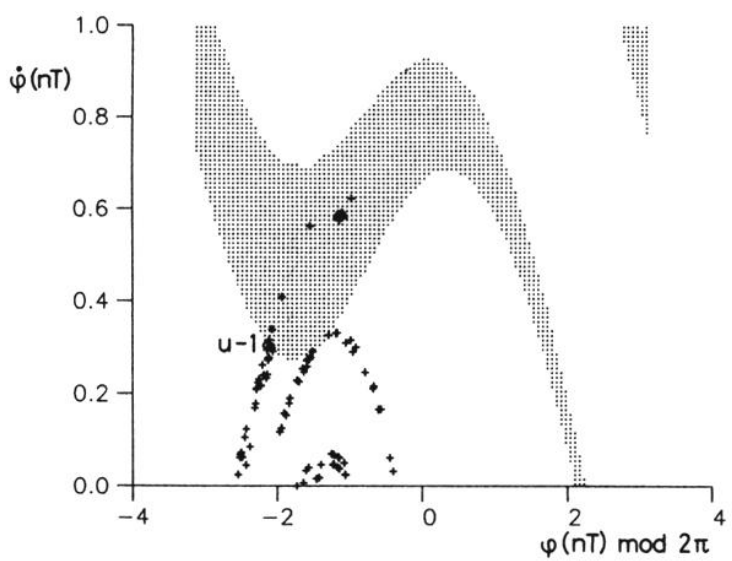

FIG. 5. Heteroclinic crossings after the boundary crisis. $\eta=1.823$, and the other parameter values used are as in Fig. 1 . Notation as in Fig. 4. 


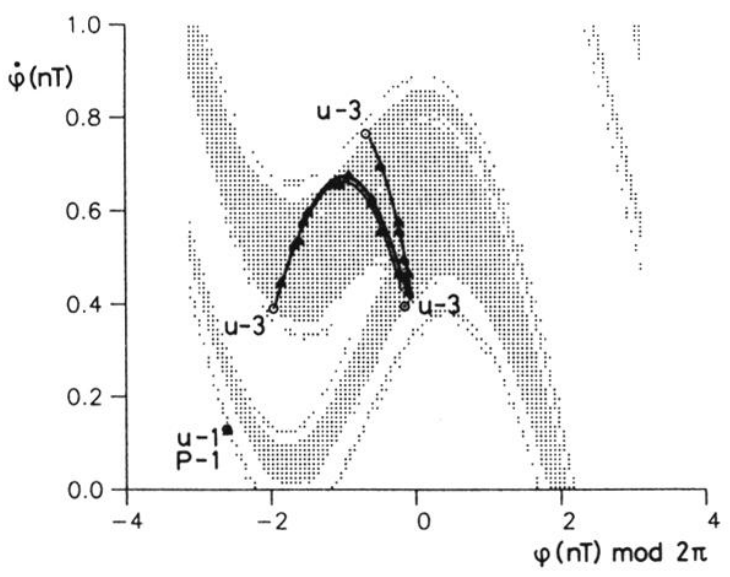

FIG. 6. Heteroclinic tangencies at the interior crisis. $\eta=1.886$, and the other parameter values used are as in Fig. 1 . Notation as in Fig. 4. 


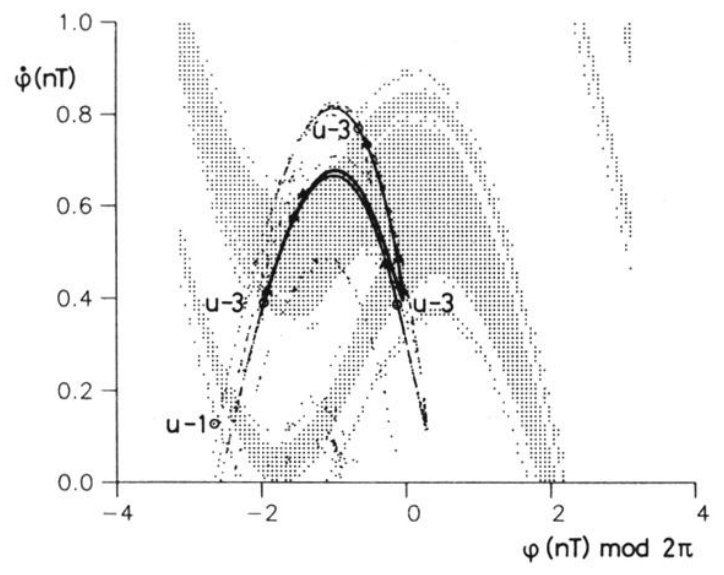

FIG. 7. Heteroclinic crossings at the interior crisis. $\eta=1.888$, and the other parameter values used are as in Fig. 1. Notation as in Fig. 4. 